\title{
Achievements of the Belgian Antibiotic Policy COORDINATION COMMITTEE (BAPCOC)
}

\author{
H Goossens (herman.goossens@uza.be) ${ }^{1}$, S Coenen², M Costers ${ }^{3}$, S De Corte ${ }^{3}$, A De Sutter ${ }^{4}$, B Gordts ${ }^{5}$, L Laurier ${ }^{6}$, M. J. Struelens ${ }^{7,8}$ \\ 1. Laboratory of Medical Microbiology, Vaccine \& Infectious Disease Institute, University of Antwerp, Antwerp, Belgium \\ 2. Department of General Practice, Vaccine \& Infectious Disease Institute, University of Antwerp, Antwerp, Belgium \\ 3. Federal Public Service Health, Food Chain Safety and Environment, Brussels, Belgium \\ 4. Department of General Practice, University of Ghent, Ghent, Belgium \\ 5. Department of Microbiology and Infection Control, St. John's General Hospital, Bruges, Belgium \\ 6. Federal Agency for Medicines and Health Products, Brussels, Belgium \\ 7. Department of Microbiology, Hôpital Erasme, Brussels, Belgium \\ 8. Infectious Diseases Epidemiology, School of Public Health, Université Libre de Bruxelles, Brussels, Belgium
}

A Belgian Antibiotic Policy Coordination Committee (BAPCOC) was officially established in 1999 by Royal Decree. The overall objective of BAPCOC is to promote judicious use of antibiotics in humans and animals and to promote infection control and hospital hygiene, with the overall aim to reduce antibiotic resistance. BAPCOC fostered strong and interdisciplinary public health, scientific and political leadership, which led to many evidence-based interventions such as multimedia campaigns to promote the prudent use of antibiotics in the community, national campaigns to promote hand hygiene in hospitals, publication of clinical practice guidelines, staffing and technical support for establishment of antibiotic management teams in all Belgian hospitals, surveillance programmes on antibiotic use and resistance in humans and animals and the promotion of research. These activities and interventions resulted in a measurable decrease in antibiotic use and resistance in the community and hospitals.

\section{Introduction}

Belgium is a small federal country with 10.5 million inhabitants living in three regions. In 1999, the Belgian Ministry of Health established by Royal Decree an official committee, called the Belgian Antibiotic Policy Coordination Committee (BAPCOC) [1].
The specific objectives of BAPCOC are to promote judicious use of antibiotics in humans and animals and enhance infection control and hospital hygiene, with the overall aim of reducing antibiotic resistance.

The specific tasks of BAPCOC are to:

- collect information on antibiotic use and resistance to antibiotics in humans and animals;

- publish reports on the evolution of antibiotic use and resistance;

- create awareness of the evolution of antibiotic resistance and the risks for public health;

- publish recommendations on the detection and surveillance of antibiotic resistance, on the appropriate use of antibiotics, on indications for prophylactic and therapeutic use of antibiotics, on the evaluation and the surveillance of antibiotic use in humans and animals, and on the implementation of international recommendations on the prudent use of antibiotics in humans and animals;

- and to publish recommendations on future research into the emergence, spread and control of antibiotic resistance.

T A B L E

Key activities of the Belgian Antibiotic Policy Coordination Committee (BAPCOC)

\begin{tabular}{|c|c|}
\hline Activity & Budget \\
\hline Multimedia campaigns to promote the prudent use of antibiotics in the community & 400,000 E per campaign \\
\hline National campaigns to promote hand hygiene in hospitals & 125,000 E per campaign \\
\hline Staffing and technical support for establishment of antibiotic management teams in all Belgian hospitals & 3.6 million $€$ each year \\
\hline Publication of clinical practice guidelines & 25,000 € per guideline \\
\hline Publication of guide to antibiotic prescribing in ambulatory care & 100,000 E per guide \\
\hline Surveillance programmes on antibiotic use and resistance in humans and animals & 100,000 E each year \\
\hline $\begin{array}{l}\text { Promotion of research e.g. } \\
\text { - prevalence of MRSA among nursing home residents } \\
\text { - prevalence of MRSA ST398 in pigs and pig farmers }\end{array}$ & $\begin{array}{l}100,000 \text { E } \\
150,000 \text { E }\end{array}$ \\
\hline Support infection control practices (better funding and clear organisation in hospitals) & 3.4 million $\mathrm{E}$ additional funding in 2007 \\
\hline
\end{tabular}


To address these specific tasks BAPCOC founded the following five multidisciplinary working groups: ambulatory care, hospital care, awareness campaigns, infection control and veterinary medicine. The working groups are composed of microbiologists, infectious diseases' and infection control specialists, epidemiologists, general practitioners (GPs), pharmacists, nurses, veterinarians, basic researchers, public health experts and health economists. The (scientific) secretariat, responsible for their day-to-day management, is hosted by the Federal Public Service Health, Food Chain Safety and Environment, Brussels, Belgium. A Steering Committee, composed of the presidents of the working groups, the chair and vice-chair of BAPCOC, meets monthly. The Steering Committee is responsible for the continuity, interaction and follow-up of initiatives and projects. At plenary meetings of BAPCOC, which are held every four months, the working groups report on their activities so that all stakeholders, including policy makers, scientific organisations, public health institutes are informed about the BAPCOC activities and results.

The key BAPCOC activities and corresponding budgets are listed in the Table. BAPCOC's annual budget in 2007 was 7.8 million EUR. Furthermore, BAPCOC participates in European projects, such as European Surveillance of Antimicrobial Consumption (ESAC; www.esac.ua.ac.be), European Antimicrobial Resistance Surveillance System (EARSS; www.rivm.nl/earss), ABS International (www.abs-international.eu), and e-bug (www.e-bug.eu).

This paper discusses selected examples of the activities and achievements of BAPCOC.

Public antibiotic awareness campaigns

The BAPCOC working group for public awareness campaigns set the following goals:

- to provide the general public with a better understanding of the natural course of minor and self-limiting infections, such as common cold, acute bronchitis, or influenza;

- to explain when the use of antibiotics is needed, i.e. in case of serious bacterial infections;

F I G U R E 1

Outpatient antibiotic use in packages per 1,000 inhabitants per day, Belgium, July 1997 to June 2007

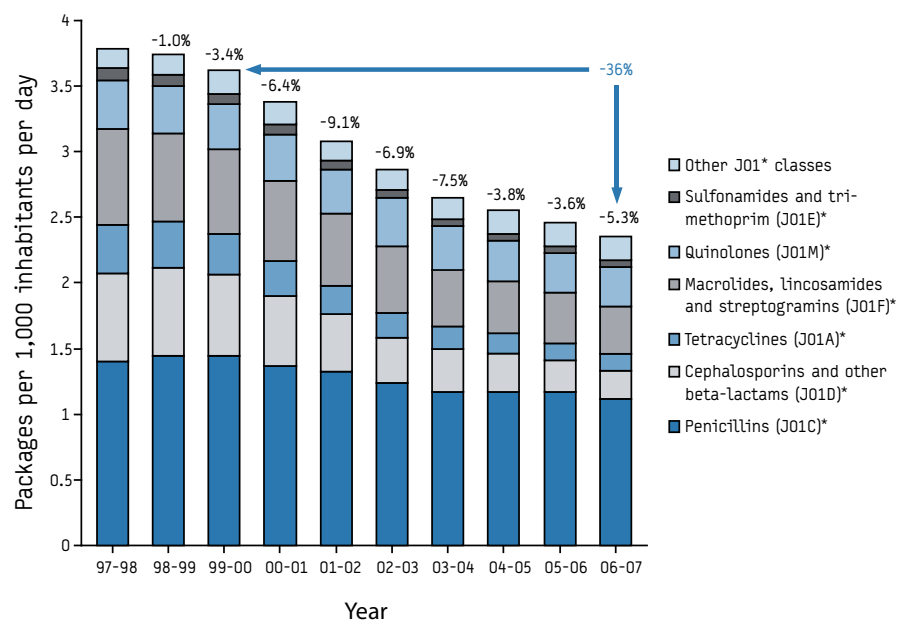

*Anatomical Therapeutic Chemical (ATC) classification code
- to underline the consequences of emergence of resistance to antibiotics;

- and to facilitate a discussion between patients doctors and pharmacists on the need for appropriate antibiotic use.

No specific target for reductions in antibiotic sales was set.

In December 2000, BAPCOC launched a media campaign which ran over three consecutive winter seasons and concentrated on simple messages that were conveyed through booklets, handouts, posters, prime-time television and radio spots, and websites, like "Use antibiotics less frequently, but better", "Save antibiotics, they may save your life", and "Talk to your doctor, talk to your pharmacist" (www.red-antibiotica.org) [2,3]. The involvement of GPs, paediatricians, pneumologists, ear, nose and throat specialists as well as of retail pharmacists was sought through personalised letters accompanied by campaign materials for presentation to patients. In November 2004, a new media campaign was launched, using the slogan "Antibiotics are ineffective for the common cold, acute bronchitis and flu"; this ran until last winter season (www. antibiotics-info.be). On 18 November 2008, a new media campaign will be launched to mark the European Antibiotic Awareness Day.

The impact of these activities has been evaluated through pre-and post-campaign face-to face interviews with the public, post-campaign surveys of the GPs, records of antibiotic sales and prescriptions in the retail pharmacies, and evolution of antibiotic resistance among pathogens frequently affecting the community. Outpatient antibiotic use, expressed by the number of reimbursed packages per 1,000 inhabitants per day, decreased by $36 \%$ between the winter season 1997-8 and 2006-7 in Belgium (Figure 1) [4]. Penicillin, tetracycline and macrolide resistance in Streptococcus pneumoniae increased up to the year 2000, after which it decreased substantially (Figure 2). Similarly, macrolide resistance in Streptococcus pyogenes decreased dramatically from $17 \%$ in 2001 to $2 \%$ in 2007 (Figure 3).

\section{National hand hygiene campaigns}

BAPCOC has organised two countrywide campaigns - in 2005 and in 2007 - for the prevention of nosocomial infections by improving

\section{F I G U R E 2}

Penicillin, tetracycline, macrolide (erythromycin) and ofloxacine resistance in Streptococcus pneumoniae, Belgium, 1985-2007

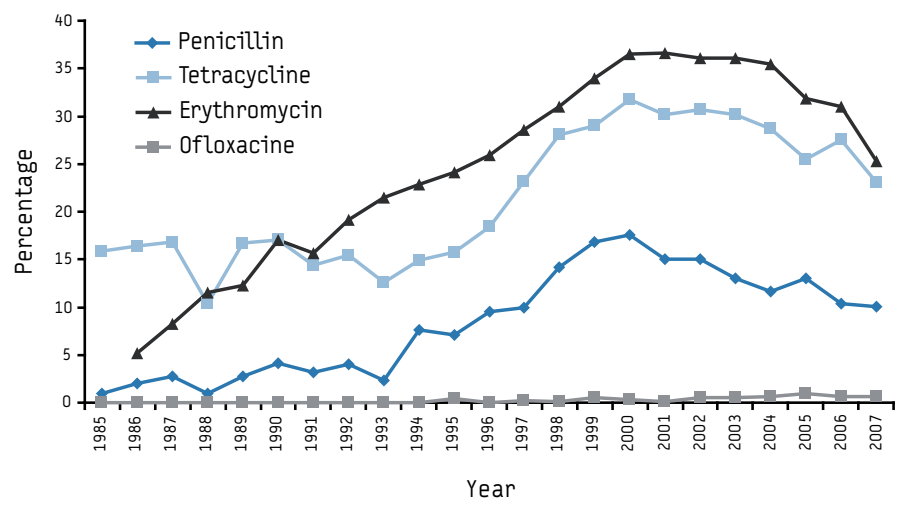

Number of strains tested varied between 1,218 in 2002 and 1,744 in 2005. Source: National Reference Centre S. pneumoniae (University of Leuven) 
hand hygiene compliance in Belgian hospitals. Key components of these campaigns were audit with performance feedback, reminders (posters), educational sessions for healthcare workers, promotion of alcohol-based hand rubs, and patient awareness (folders). Participation, on a voluntary basis, was excellent for both campaigns: $97 \%$ for acute care hospitals, $66 \%$ for long-term care hospitals and $63 \%$ for psychiatric hospitals. Overall compliance with hand hygiene (measured by direct observation) increased significantly from $49 \%$ to $69 \%$ for the first campaign and from $53 \%$ to $69 \%$ for the second campaign. The third campaign will be held in December of 2008.

\section{Antibiotic Management Teams in hospitals}

Since the 1990s, there has been a move in Belgian hospitals to establish multidisciplinary antibiotic management teams (AMT) to contain antibiotic resistance and improve antibiotic prescribing. The BAPCOC working group on hospital care developed an implementation plan for this strategy by mobilising federal funding for AMTs and antibiotic managers. In 2002, a pilot project started with a yearly budget of 0.93 million euros for hiring trained antibiotic managers in 37 hospitals, selected from 69 candidate hospitals for their expertise and experience with a local antibiotic stewardship programme. Based on successful activity reports of these pilot hospitals [5], the project was extended to 61 hospitals in 2006 by doubling the financial support to 1.83 million euros and since July 2007 to all acute care hospitals with an annual budget of 3.61 million euros. The AMTs will remain in the BAPCOC programme and their tasks have been defined by Royal Decree. BAPCOC provides scientific support to the participating hospitals by means of a dedicated training course, national study days, standardised evaluation of local progress reports and national surveillance of antibiotic consumption in hospitals. In the years 2002-7, over 600 hospital pharmacists and physicians participated in BAPCOC-supported interuniversity teaching courses in antibiotic management. National workshops were held twice to share good practices between hospital AMTs. Analysis of the recent reports of the 61 pilot phase hospitals clearly demonstrates general adoption of well- established quality improvement interventions, such as an antibiotic formulary, guidelines for prophylactic and therapeutic antibiotic use, regular analysis of local antibiotic consumption and resistance profiles, and increasing conductance of clinical

\section{F I G U R E 3}

Macrolide (erythromycin) resistance in Streptococcus pyogenes, Belgium, 2002 to 2007

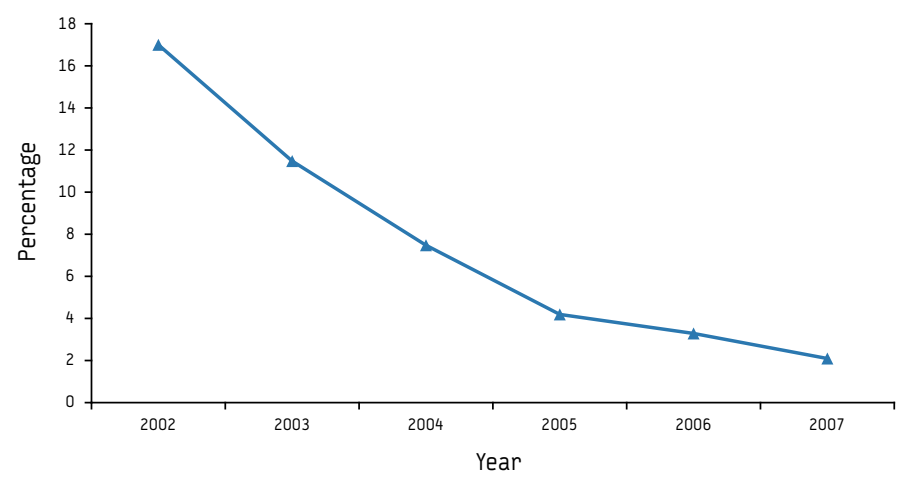

Number of strains tested varied between 1298 in 2002 and 934 in 2007. Source: National Reference Centre Streptococcus pyogenes (University of Antwerp) audits and drug use evaluations. In a recent international survey of structural indicators of antibiotic stewardship programmes, Belgian hospitals scored high on average (3.75/5). The survey showed that $90 \%$ of hospitals had key structural resources and tools in place for effective antibiotic management and infection control [6].

The impact of these activities in hospital infection control and antibiotic stewardship is monitored through a newly developed surveillance of hospital-anti-infective drug consumption as well as by several longstanding surveillance schemes of nosocomial infection and multidrug resistant pathogens coordinated by the Scientific Institute of Public Health. These include methicillinresistant Staphylococcus aureus (MRSA), extended spectrum betalactamase (ESBL)-producing Enterobacteriaceae and pathogenic Clostridium difficile. An example of encouraging results is the 35 $\%$ relative reduction since 2004 in the incidence of nosocomial acquisition of MRSA among patients admitted to acute care hospitals [7].

\section{Publication of guidelines and a guide for appropriate use of} antibiotics

BAPCOC, in collaboration with scientific experts and organisations, produced evidence-based guidelines for the appropriate use of antibiotics in hospitals and ambulatory care for important infectious disease syndromes such as acute sore throat, otitis media, sinusitis, community-acquired pneumonia, uncomplicated and complicated urinary tract infections. All BAPCOC guidelines were disseminated free of charge to all relevant physicians (GPs and/or specialists) in Belgium. The guideline recommendations for ambulatory care were supplemented by conclusions of systematic literature reviews. BAPCOC also produced a booklet in 2004 on antibiotic treatment of community-acquired infectious diseases. All Belgian GPs received a copy of this first antibiotic booklet in 2004. A copy of the second edition of this antibiotic guide will be distributed among all primary care physicians in November 2008. The impact of this antibiotic guide for outpatients is monitored based on antibiotic prescribing in the community by indication, age and class of antibiotics.

Surveillance programmes on antibiotic use and resistance in humans and animals

BAPCOC provides support and additional funding for surveillance of MRSA, vancomycin-resistant enterococci (VRE), ESBL-producing Enterobacteriaceae, S. pneumoniae and S. pyogenes. BAPCOC also supports surveillance programmes on antibiotic use in hospitals, such as point prevalence surveys. The results of these surveillance programmes are published in an annual report, published by the Public Health Institute in Brussels.

\section{Research projects funded by BAPCOC}

BAPCOC funded a number of research projects to provide scientific evidence for national policies and guidelines of which examples are given below.

\section{National survey of prevalence of MRSA carriage among residents of nursing homes}

BAPCOC funded the first national survey of prevalence of MRSA carriage among residents of nursing homes in Belgium. The objectives of this survey were to study the national prevalence of MRSA colonisation in Belgian nursing home residents, to identify risk factors for MRSA carriage among nursing home residents, in respect of both the individual resident and of the nursing home 
facility, and to study the molecular epidemiology and susceptibility of MRSA strains isolated from residents living in these facilities. Based on a representative sample of 3,000 residents in 60 institutions, a prevalence of $19 \%$ carriage was noted (unpublished data). Importantly, $90 \%$ of MRSA carriers found in the survey were not identified as such by the nursing and medical personnel in spite of routine MRSA screening on transfer from hospitals and a discharge letter being sent by the hospital, in the majority of cases, to the participating nursing homes at the time of patient referral. A better understanding of the MRSA reservoir in nursing homes and analysis of risk factors permitted the adaptation of an MRSA control policy. National guidelines to prevent the spread of MRSA in nursing homes have been developed and the impact of these guidelines on the evolution of MRSA carriage in nursing homes will be evaluated by a new prevalence survey in the near future.

\section{Prevalence survey of MRSA in swine farmers in Belgium}

BAPCOC funded a survey on MRSA in swine farmers and their household contacts, to determine if MRSA strains in those farmers are related to those in swine, to characterise and compare phenotypes and genotypes of MRSA strains from humans and swine, and to study risk factors for MRSA colonisation and assess levels of personal hygiene in farm workers. Extensive colonisation with two subtypes of the livestock-associated ST398 MRSA strain was found in swine (44\% carriers from $68 \%$ of farms) and farmers $(38 \%)$ in contact with swine and other animals. Reported use of personal protection equipment and decontamination showed no difference in the rates of colonisation [8]. As a result of this survey, a National MRSA Med Vet Task Force was established to coordinate further investigation of the epidemiology of MRSA in animals and persons in contact with animals and to develop guidelines for risk management.

National investigation on the infection control practices in surgery

BAPCOC funded and supported a national investigation on infection control practices in operating rooms. More than half of all acute care hospitals participated in a national inventory evaluating the extent to which internationally suggested infection prevention precautions are defined, carried out and followed-up. A list of essential precautions was grouped into different categories: architecture and structure, environmental cleaning, peri-operative procedures, sterilisation, logistic activities and surveillance of postoperative wound infections.

For each essential item, participants reported the estimated degree of compliance in their institution, the existence of a standard operating procedure (SOP) for the performance for this precaution, and finally whether the respective items are written down in institutional guidelines or procedures. The investigation clearly demonstrated the extensive variability in infection control practices in Belgian operating rooms, with respect to standards described in institutional operating procedures as well as to actual compliance with local and/or (inter)national precaution measures. The results are detailed in an advisory document reported to the authorities, stating the necessity of quality control standards implementation and official follow-up of procedures regarding perioperative infection control.

\section{Conclusions}

Our experience demonstrates that strong joint and interdisciplinary public health, scientific and political engagement in Belgium led to many evidence-based interventions, aimed at both the general public and healthcare professionals and those interventions in return resulted in a decrease in antibiotic use and resistance in the community and hospitals. They also show that creating awareness for the factors driving antimicrobial resistance and providing a knowledge base for physicians, public health experts and scientists is crucial in containing antibiotic resistance. A number of scientific conferences and public health workshops organised by BAPCOC were helpful in this respect.

\section{References}

1. Royal Decree of April 26, 1999. Creation of Belgian Antibiotic Coordination Committee (BAPCOC). Belgisch Staatsblad July 31, 1999.

2. Goossens H, Guillemot D, Ferech M, Schlemmer B, Costers M, van Breda M, et al. National campaigns to improve antibiotic use. Eur J Clin Pharmacol. 2006; 62(5):373-9.

3. Bauraind I, Lopez-Lozano JM, Beyaert A, Marchal JL, Seys B, Yane F, et al. Association between antibiotic sales and public campaigns for their appropriate use. JAMA. 2004;292(20):2468-70.

4. Davey P, Ferech M, Ansari F, Muller A, Goossens H, on behalf of the ESAC Project Group. Outpatient antibiotic use in the four administrations of the UK: cross-sectional and longitudinal analysis. J Antimicrob. Chemother. 2008 Sep 11 [Epub ahead of print].

5. Sourdeau L, Struelens MJ, Peetermans WE, Costers M, Suetens C. Hospital Care Working Group of Belgian Antibiotic Policy Coordination Committee (BAPCOC). Implementation of antibiotic management teams in Belgian hospitals. Acta Clin Belg 2006; 61(2):58-63.

6. Struelens MJ, Costers M. Belgian Antibiotic Policy Coordination Committee (BAPCOC) - Hospital Care Working Group

7. Hospital antibiotic management in Belgium- results of the ABS maturity survey of the ABS International group. Wien Klin Wochenschr 2008;120(9):284-8.

8. Jans B, Struelens M, [Surveillance des MRSA dans les hôpitaux aigus belges : premier semestre 2007]. Surveillance of MRSA in acute-care Belgian hospitals: first quarter 2007. Brussels: Institut Scientifique de Santé Publique 20007. Available from: http://www.iph.fgov.be/nsih/surv_mrsa/download_fr.asp

9. Denis 0, Suetens C, Hallin M, Ramboer I, Catry B, Gordts B, et al. High prevalence of "livestock-associated" methicillin-resistant Staphylococcus aureus ST398 in swine and pig farmers in Belgium. In: Abstracts of the 18th European Congress of Clinical Microbiology and Infectious Diseases, (ECCMID), Barcelona, 19-22 April 2008.

This article was published on 13 November 2008.

Citation style for this article: Goossens H, Coenen S, Costers M, De Corte S, De Sutter A, Gordts B, Laurier L, Struelens MJ. Achievements of the Beloian Antibiotic Policy , Gordination Committe (BAPCOC). Euro Surveill. 2008:13(46):pii=19036. Available online: http://www.eurosurveillance.org/ViewArticle.aspx?ArticleId=19036 